\title{
REAÇÃO DE MAILLARD: UMA REVISÃO
}

\section{Maillard Reaction: review}

\begin{abstract}
Júlia d'Almeida Francisquini', Evandro Martins ${ }^{1}$, Paulo Henrique Fonseca Silva ${ }^{2}$, Pierre Schuck ${ }^{3}$, Ítalo Tuler Perrone ${ }^{{ }^{*}}$, Antônio Fernandes Carvalho ${ }^{l}$
\end{abstract}

\begin{abstract}
RESUMO
A reação de Maillard é um importante tema de estudo em ciência e tecnologia de alimentos e diferentes áreas do conhecimento estão integradas como química, engenharia de alimentos, nutrição e tecnologia de alimentos. O objetivo deste artigo é apresentar os conceitos envolvidos na reação de Maillard levando em conta as etapas da reação, os principais produtos e algumas consequências tecnológicas para os produtos lácteos.
\end{abstract}

Palavras-chave: glicosilamina; produtos de Amadori; 5-hidroximetilfurfural; glicação.

\begin{abstract}
Maillard reaction is an important subject of study in food science and technology and different areas of knowledge are involved such as chemistry, food engineering, nutrition and food technology. The objective of this paper is to present the basic concepts of the Maillard reaction, such as the reaction stages, the main compounds producced and some technological consequences for dairy products.

Keywords: glucosamine, products of Amadori, 5-hydroxymethylfurfural, glycation.
\end{abstract}

1 Universidade Federal de Viçosa (UFV), Av. Peter Henry Rolfs, s/n, Campus Universitário, 36570-900, Viçosa, MG, Brasil. Email: italotulerperrone@gmail.com

2 Universidade Federal de Juiz de Fora (UFJF), Juiz de Fora, MG, Brasil.

3 UMR STLO, INRA, Agrocampus-Ouest, França.

* Autor para correspondência.

Recebido / Received: 16/11/2016

Aprovado / Approved: 01/06/2017 


\section{INTRODUÇÃO}

Existem diferentes tipos de escurecimento dos alimentos os quais ocorrem rotineiramente tanto nas residências quanto na indústria. $\mathrm{O}$ escurecimento enzimático corresponde àquele que resulta em descoloração oriunda de reações catalisadas por uma enzima conhecida por polifenol oxidase (PPO). Tal enzima age principalmente sobre frutas e vegetais gerando consequências indesejáveis como perdas econômicas, diminuição do valor nutritivo, alterações de sabor e aparência do alimento ou desejáveis como cor, sabor e aroma no caso de alimentos como chá, café, cacau e ameixa seca (FENNEMA, 2010). Há também o escurecimento não-enzimático, mais lento do que o escurecimento enzimático por não ter a enzima catalisadora da reação, sendo representado pelas reações de caramelização, Maillard e de oxidação do ácido ascórbico (vitamina C). Cada alimento, de acordo com suas características, vai apresentar um escurecimento específico, já que a velocidade da reação é dependente da natureza dos componentes reativos dos alimentos (BRIÃO et al., 2011).

Dentre as reações de escurecimento nãoenzimático destaca-se a reação de Maillard (RM) que foi descoberta em 1912 por LouisCamille Maillard durante a tentativa da síntese de peptídeo em condições fisiológicas. É objeto de grande interesse na atualidade, estando relacionado com aspectos químicos, sensoriais, nutricionais, toxicológicos e manifestações in vivo (BASTOS et al., 2011; MEHTA; DEETH, 2016).

A RM é representada por uma complexa cascata de reações, que surge principalmente durante o aquecimento e armazenamento prolongado de produtos alimentícios resultando em modificações na qualidade dos alimentos, favorecendo a formação de compostos responsáveis pelo aroma, sabor e cor dos alimentos tratados termicamente. Esta reação é subdividida em três estágios: estágio inicial, estágio intermediário e estágio final (BASTOS et al., 2011; BRIÃO et al., 2011; FENNEMA, 2010; MEHTA; DEETH, 2016; RODRIGUEZ, et al., 2016).

O estágio inicial consiste na condensação do grupo carbonila do açúcar redutor com o grupamento amino livre de aminoácidos, peptídeos ou proteínas, o que ocorre através do ataque nucleofílico do par de elétrons do nitrogênio do grupo amino, levando ao início da reação. Como consequência desta condensação há formação da base de Schiff instável que libera água e forma uma glicosilamina. Assim, esta base de Schiff sofre rearranjos sequenciais produzindo uma aminocetose razoavelmente estável conhecida como produto de Amadori (açúcar aldose) ou produto de Heyns (açúcar cetose). Estes produtos, desenvolvidos no estágio inicial, são estáveis e não possuem cor, fluorescência ou absorção na região ultravioleta resultando em uma enorme variedade de produtos em distintas proporções (BASTOS et al., 2011; BRIÃO et al., 2011; FENNEMA, 2010; MEHTA e DEETH, 2016).

Com o prolongamento do aquecimento ou armazenamento inicia-se a segunda fase. Os produtos de Amadori ou produtos de Heyns são fragmentados e originam uma série de reações como desidratação, enolização e retroaldolização. Nesta etapa intermediária, surgem os compostos dicarbonílicos, redutonas, derivados do furfural e produtos da degradação de Strecker, podendo ocorrer o aparecimento de um derivado furano que origina uma hexose comumente conhecida por 5-hidroximetilfurfural (BASTOS et al., 2011; BRIÃO et al., 2011; FENNEMA, 2010; MEHTA; DEETH, 2016).

Os compostos originados na fase intermediária são fluorescentes e com capacidade de absorção da radiação na região ultravioleta. Os mesmos são cíclicos e altamente reativos, se polimerizando junto com resíduos 
de lisina ou arginina em proteínas, resultando em compostos estáveis e culminando na formação de pigmentos escuros conhecidos como melanoidinas. Estes pigmentos levam à coloração dos alimentos sendo desejáveis ou indesejáveis e fazendo parte do estágio final da RM (BASTOS et al., 2011; BRIÃO et al., 2011; FENNEMA, 2010; LIU et al., 2014).

O controle da formação de produtos de Amadori é o ponto chave para limitar a intensidade da RM em lácteos. De acordo com Troise et al. (2016), o emprego da frutosamina oxidase (Faox) em leites UHT baixa lactose $(<0,1 \%$ de lactose $)$ reduziu a velocidade da RM, assim como, a adição de flavonóides (epicatequinas - EC eepicatequinas gallate ECG) foi efetiva no controle da RM durante o processamento e estocagem de leite UHT (SCHAMBERGER; LABUZA, 2007).
As diferentes etapas da RM em alimentos são representadas na Figura 1.

A reação de Maillard pode ser afetada por diversos fatores entre eles a temperatura e o $\mathrm{pH}$. A velocidade desta reação é lenta a temperaturas mais baixas e praticamente duplica a cada aumento de $10{ }^{\circ} \mathrm{C}$ entre $40^{\circ} \mathrm{C}$ e $70^{\circ} \mathrm{C}$. Em soro em pó o valor de $\mathrm{Q}_{10}$ varia entre 1,77 e 4,14, enquanto que a magnitude da energia de ativação encontra-se entre 15,9 e 28,4 kcal/mol (SITHOLE et al., 2005; ARENA et al., 2017; RODRIGUEZ, et al., 2016).

O pH também exerce efeito sobre a intensidade da reação, assim a velocidade máxima ocorre na faixa alcalina entre $\mathrm{pH} 9 \mathrm{e}$ 10 (BASTOS et al., 2011; FENNEMA, 2010; RODRIGUEZ et al., 2016).

O tipo de amina influencia diretamente

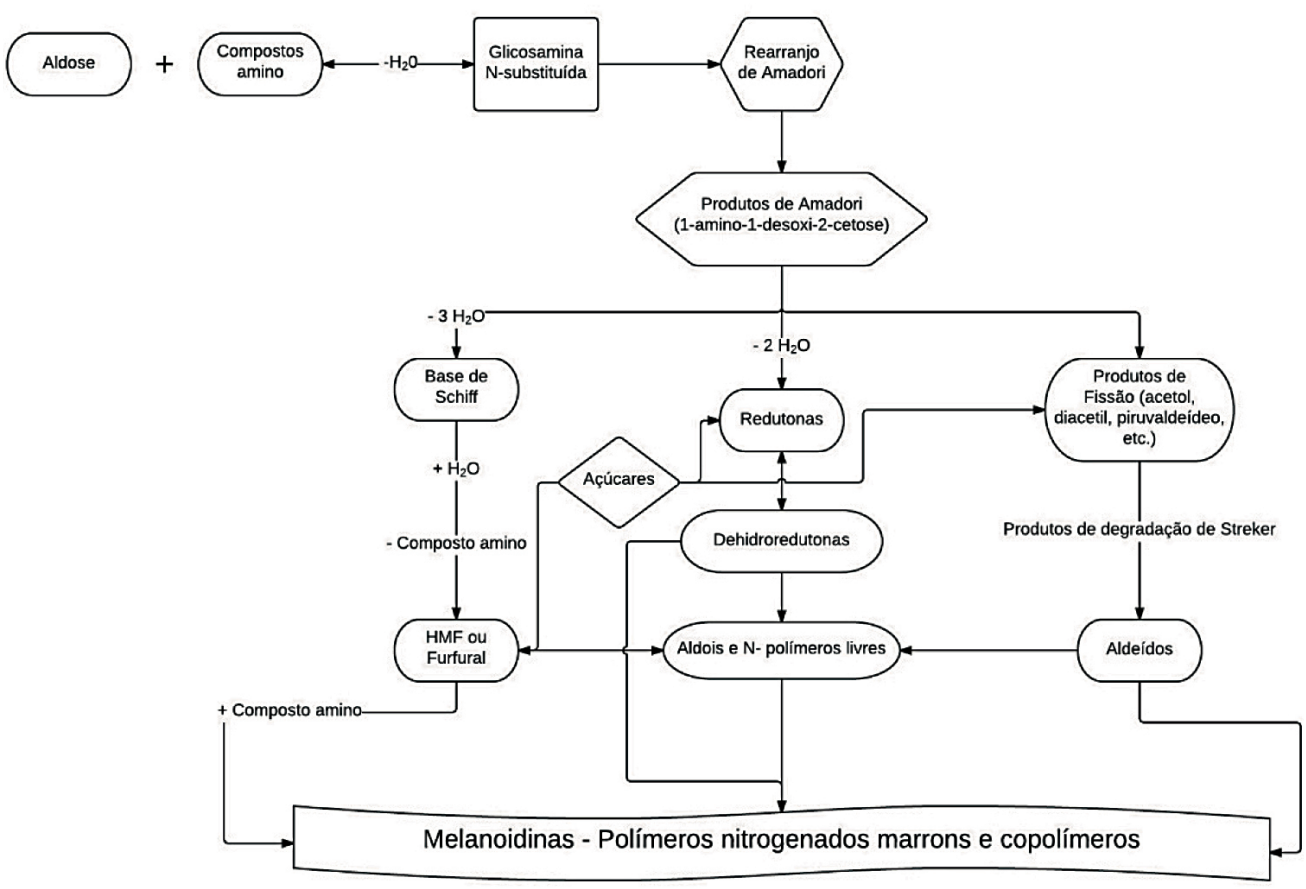

Figura 1 - Representação das principais etapas da reação de Maillard em alimentos.

Fonte: adaptado de Arena et al. (2017); McSweeney; Fox (2009). 
na facilidade de desenvolvimento da RM. Os aminoácidos de alta reatividade representados pela lisina, glicina, triptofano, tirosina facilitam a ocorrência da reação quando comparados com os de reatividade média como prolina, leucina, isoleucina, hidroxiprolina, metionina e com os de fraca reatividade como histidina, treonina, ácido aspártico, ácido glutâmico e cisteína. A lisina, por apresentar o grupo amino épsilon livre, apresenta alta reatividade sendo mais susceptível à reação (carbonila-amino) o que pode reduzir o valor nutricional do alimento em que se encontra (BASTOS et al., 2011; FENNEMA, 2010; RODRIGUEZ et al., 2016).

O açúcar redutor é essencial para que a reação de Maillard aconteça, as pentoses são mais reativas que as hexoses que são mais reativas que os dissacarídeos. Este tipo de escurecimento sucede com maior ênfase nos valores intermediários de atividade de água $(0,5$ e 0,8$)$. Isto porque em baixa atividade de água $(\cong 0,20)$ a velocidade tende a zero por uma diminuição de solvente e em altos valores $(\cong 0,9)$ os reagentes se encontram muito diluídos o que diminui a velocidade de escurecimento (BASTOS et al., 2011; FENNEMA, 2010; RODRIGUEZ et al., 2016). A magnitude da atividade de água em produtos lácteos desidratados é um atributo de controle do processo de desidratação, sendo alcançado por meio da relação de equilíbrio entre a umidade relativa do ar de saída do spray dryer e o produto (SCHUCK et al., 2016; O'CALLAGHAN; HOGAN, 2013). A RM e as consequentes interligações das proteínas acarretam perda da solubilidade de produtos lacteos desidratados, em especial os concentrados proteicos do leite, com $80 \%$ de proteínas (LE et al., 2013).

Além destes fatores, os íons metálicos (ferro e cobre), luz, sulfito, condições de armazenagem, tipo, tempo e temperatura do tratamento térmico e método de cozimento ou concentração dos alimentos podem ser consi- derados interferentes nesta reação (BASTOS et al., 2011; FENNEMA, 2010).

A extensão da reação de Maillard pode ser monitorada pelo surgimento de compostos os quais permitem avaliar a intensidade do processamento térmico aplicado bem como as prováveis alterações nutricionais relacionadas a ele. Entre estes compostos estão: a furosina, o hidroximetilfurfural e a carboximetilisina (LIU et al., 2014; MEHTA; DEETH, 2016; RAJCHL et al., 2013).

\section{REFERENCIAL TEÓRICO}

\section{Produtos da reação de Maillard (PRM)}

Os PRM são consumidos diariamente pela população, por meio da ingestão de alimentos como leite, produtos de panificação, cereais infantis e matinais, caramelo, mel, café, cerveja, chocolate, carnes, vegetais desidratados e frutas processadas apresentando um importante papel tecnológico e sensorial para a indústria de alimentos por suas propriedades antioxidantes, flavor, aroma, odor, textura e coloração (BASTOS et al., 2011; HELLWIG et al., 2015; VHANGANI; WYK, 2016).

Em geral, alimentos ricos em lipídeos e que são submetidos a calor seco como grelhados, fritos e assados apresentam os maiores teores de produtos da reação de Maillard. Métodos mais brandos de aquecimento como ensopados e a vapor apresentam menores teores de produtos da RM (BASTOS et al., 2011; HELLWIG et al., 2015).

Sabe-se que os PRM correspondem a um grupo heterogêneo de substâncias de baixo peso molecular. O HMF é considerado um destes produtos e não existe em alimentos crus e frescos, porém é produzido rapidamente durante o processamento térmico e no armazenamento prolongado, principalmente em alimentos ricos em carboidratos e lipídeos (RAJCHL et al., 2013; ROSATELLA et al., 2011). 
O 5-hidroximetilfurfural foi relatado pela primeira vez no final do século 19 , a partir da descrição da sua síntese por aquecimento da inulina com uma solução de ácido oxálico sob pressão (ROSATELLA et al., 2011).

Em temperaturas acima de $100{ }^{\circ} \mathrm{C}$ os produtos de enolização que são formados durante o estágio intermediário da reação de Maillard, sofrem desidratação com fragmentação do açúcar e degradação de aminoácidos. A desidratação do açúcar neste caso, pode acontecer de duas formas dependentes do
pH. Em condições ácidas, com pH menor ou igual a 7, há formação desta hexose nomeada pela União Internacional de Química Pura e Aplicada (IUPAC) de 5-(hidroximetil) furan-2-carbaldeído. A mesma também é conhecida por 5-hidroximetilfurfural ou 5-hidroxi-2-metilfuraldeido ou hidroximetilfurfural ou 5-hidroximetilfuraldeído (KAVOUSI et al., 2015; NCBI, 2005). A Figura 2 representa as etapas da reação de Maillard na qual forma-se o HMF.

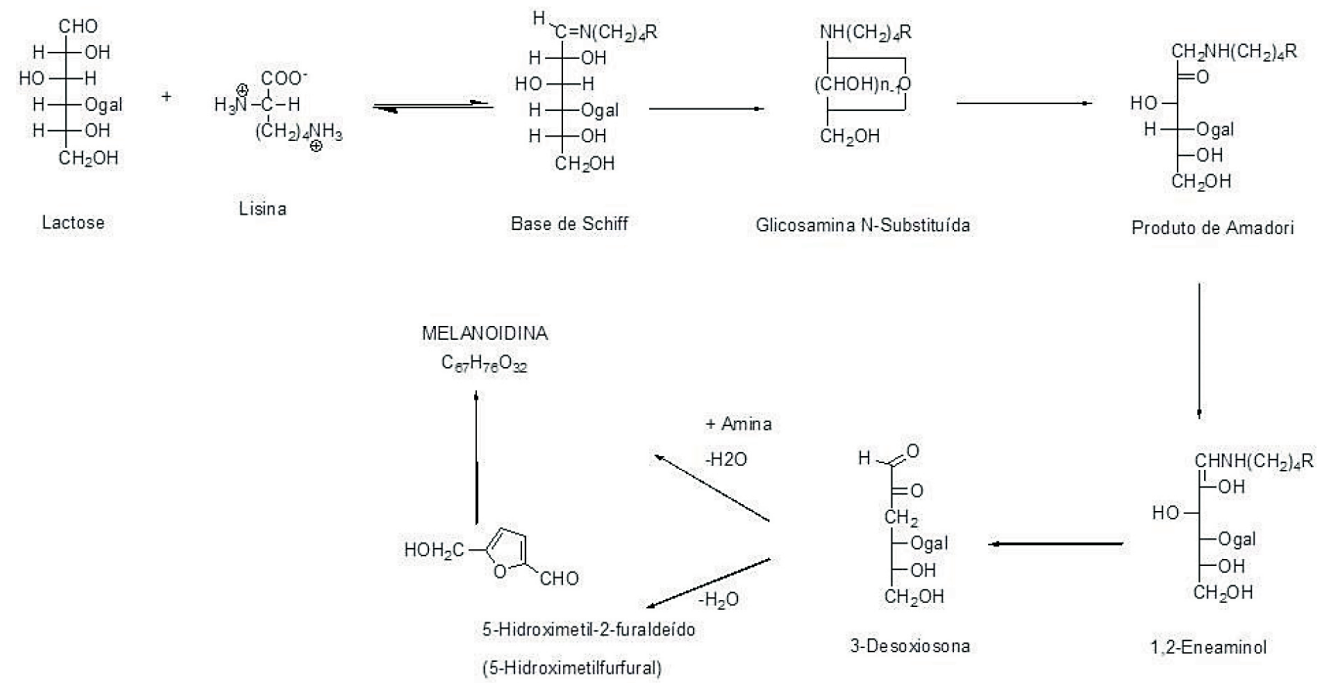

Figura 2 - Representação das etapas da reação de Maillard destacando o HMF

Fonte: adaptado de Arena et al. (2017), Fennema et al. (2010), Mehta; Deeth (2016).
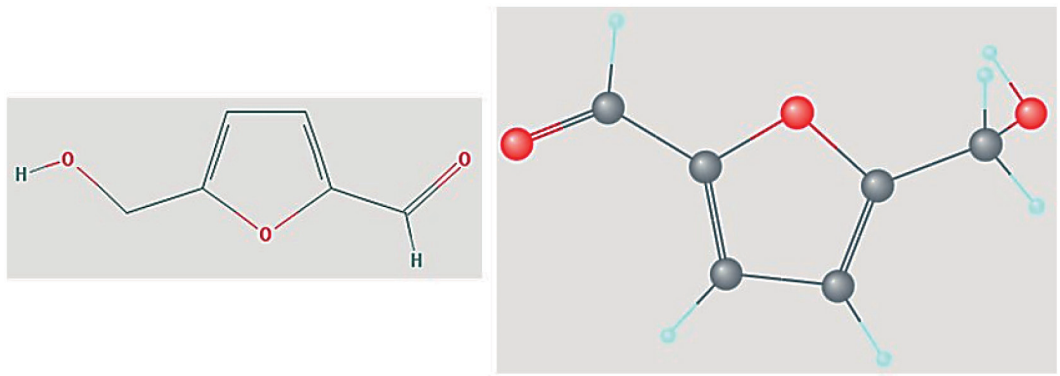

Figura 3 - Fórmulas estruturais do 5-hidroximetilfurfural, NCBI (2005) 
Tabela 1 - Propriedades físicas, químicas e experimentais do 5-hidroximetilfurfural

\begin{tabular}{c|c}
\hline Propriedades & Característica do HMF \\
\hline Peso molecular & $126,11 \mathrm{~g} \mathrm{~mol}^{-1}$ \\
Coloração & Sólido cristalino \\
Odor & Flores de camomila \\
Manteiga, caramelo \\
Textura & ou mofado \\
Ponto de fusão & $31,5^{\circ} \mathrm{C}$ \\
Densidade & $1,2062 \mathrm{~g} / \mathrm{mL}$ \\
Pressão de vapor & a $25^{\circ} \mathrm{C}$ \\
& $5,2810^{-3} \mathrm{mmHg}$ \\
\hline
\end{tabular}

Fonte: Elaborado pelo autor a partir de NCBI (2005).

O HMF é cíclico e pode ser um marcador da fase intermediária da reação de Maillard sendo reativo e dependente da severidade do tratamento térmico empregado no alimento e podendo se polimerizar com grupos amino originando materiais insolúveis, de maior massa molar, que contém nitrogênio e coloração que pode variar de marrom a preto conhecidas como melanoidinas (ARENA et al., 2017; FENNEMA, 2010). $\mathrm{O}$ índice de HMF pode ser satisfatoriamente correlacionado a atributos tecnológicos e de textura do doce de leite (FRANCISQUINI et al. 2016), contudo não se mostra um índice adequado para descrever a reação de Maillard no soro em pó quando submetido a diferentes condições de armazenamento (LEIVA et al., 2017). Segundo Guerra-Hernández et al. (2002), os índices de HMF total, HMF livre, bem como, lactulose e percentual de perda de lisina mostraram-se indicadores aplicáveis ao acompanhamento do escurecimento não enzimático em alimentos infantis (produtos de base láctea destinados a alimentação infantil, também conhecidos como leites maternizados).

As melanoidinas apresentam efeitos biológicos positivos por suas atividades antioxidantes, benefícios prebióticos, função anti-hipertensiva, atividade bacteriostática, prevenção de doenças dentárias, redução do risco de câncer, ação antiinflamatória (ANDRADE, 2014; SAKKAS, 2014). Como um exemplo de ação favorável da RM encontra-se o aumento da capacidade antioxidante em isolados de proteínas do soro (LIU et al., 2014).

Lechner (1982), citado por Pinto e Wolfschoon-Pombo (1984), assegura que a denominação teor de HMF para aqueles que utilizam a metodologia de Kenney e Basete (1959) não é adequada, sendo assim o mesmo autor sugere a utilização do termo: "valor de HMF" ou "índice de ácido tiobarbitúrico". Neste trabalho o termo utilizado será índice de HMF.

Percebe-se que as gerações atuais começam a ingerir alimentos ricos em produtos da reação de Maillard cada vez mais cedo. As fórmulas infantis e alimentos de fácil preparo como fast food, frituras e industrializados muitas vezes apresentam altas concentrações dos PRM (ANDRADE, 2014; BASTOS et al., 2011). Os produtos da reação de Maillard presentes nas dietas podem elevar os níveis séricos dos produtos finais de glicação avançada (AGEs) estando diretamente relacionados com aumento do estresse oxidativo, tais como disfunções orgânicas e aumento dos níveis de moduladores inflamatórios (VLASSARA et al., 2008; KELLOW; COUGHLAN, 2015).

Um dos pontos negativos mais evidentes do escurecimento dos alimentos e dos produtos formados a partir dele corresponde à destruição ou redução do valor nutricional de aminoácidos essenciais como arginina, lisina, metionina e triptofano, além de redução da digestibilidade do alimento (MEHTA; DEETH, 2016). Além disto, o HMF pode estar relacionado com danos ao DNA, formação de inibidores do crescimento e atividades citotóxicas, genotóxicas, mutagênicas e 
carcinogênicas (MONARO, 2012; KAVOUSI et al., 2015). Na tecnologia de produção do doce de leite, a substituição de $10 \%$ da sacarose por glicose acarreta em um aumento de $90 \%$ da glicação da lisina, enquanto que a hidrólise da lactose leva a obtenção de um doce com teor de lisina livre muito baixo $(<50 \mathrm{mg}$ de lisina por g de proteína) (MALEC et al.2005).

\section{Reação de Maillard in vivo (glicação)}

A reação de Maillard também pode acontecer in vivo tornando-se conhecida como glicação, e ocorre nos tecidos e corpos fluidos estando normalmente associada a condições patofisiológicas, envolvendo a formação dos produtos finais de glicação avançada (URIBARRI et al., 2010; VISTOLI et al., 2013).

Os AGEs, também conhecidos como glicotoxinas, apresentam estruturas variadas, sendo dependentes das características individuais, do tipo de alimento ingerido e do método do tratamento térmico empregado. Os mesmos surgem pela reação de Maillard ou pela glicação as quais são iniciadas pela associação entre açúcares redutores e grupos amino livres de proteínas, lipídios ou ácidos nucleicos (POULSEN et al., 2013; URIBARRI et al., 2010).

Os AGEs são originados endogenamente, fazendo parte do metabolismo normal, podendo neste caso prevenir doenças como diabetes tipo I, além de facilitar a proliferação da intima após angioplastia, melhorar a sensibilidade à insulina, auxiliar na cicatrização de feridas. Porém, se os níveis de AGEs forem excessivamente elevados, como em condições de hiperglicemia ou estresse oxidativo, podem alcançar os tecidos e a circulação tornando-se patogênicos (POULSEN et al., 2013; URIBARRI et al., 2010; VHANGANI; WYK, 2016; VISTOLI et al., 2013).
Em adição aos produtos finais de glicação avançada que se formam no interior do corpo, existem os provenientes dos alimentos. A formação dos AGEs endógenos sucede em baixas temperaturas quando comparados com os dietéticos, desta forma existe uma menor quantidade de compostos formados in vivo quando comparado com aqueles formados nos alimentos. A quantidade de AGEs ingeridos normalmente é maior do que a quantidade formada no plasma sanguineo ou nos tecidos. As duas fontes principais, peculiares ao estilo de vida moderno, que fornecem uma oferta crescente de AGEs exógenos, são a alimentação e o tabagismo, sendo que da fração total absorvida cerca de dois terços são retidos no organismo e apenas um terço é excretado pela urina (POULSEN et al., 2013; VLASSARA et al., 2008).

O método de preparo e a composição de nutrientes do alimento podem influenciar na maior ou menor quantidade de AGEs. Alimentos submetidos a altas temperaturas e com baixo teor de umidade como grelhados, assados, tostados, secos e fritos ou alimentos ricos em lipideos/proteínas ou ainda alimentos processados por calor seco como chips, cockies, bolachas, são normalmente aqueles que apresentam maior teor destes compostos (KELLOW; COUGHLAN, 2015; URIBARRI et al., 2010; ).

Normalmente o grupo de alimentos que contém menor quantidade de AGEs são aqueles que apresentam em sua constituição carboidratos como os amidos, as frutas, os legumes e o leite, provavelmente esta situação acontece pelo maior teor de água, maior nível de antioxidantes e vitaminas ou pelo fato de que nesta categoria de alimentos, a maioria dos polissacarídeos consistem em açúcares não redutores (KELLOW; COUGHLAN, 2015; URIBARRI et al., 2010).

De acordo com a pirâmide alimentar atual o grupo dos cereais, pães, tubérculos, 
raízes e massas são os alimentos que devem ser ingeridos em maior quantidade durante o dia (BARBOSA et al., 2005). Isto pode resultar em uma maior ingestão de produtos da reação de Maillard ou AGEs e consequentemente afetar o pool endógeno, auxiliando para o excesso destes produtos no organismo modificando suas características positivas para negativas.

\section{CONSIDERAÇÕES FINAIS}

Há mais de 100 anos estuda-se a reação de Maillard e o controle de seus pontos positivos e negativos continua desafiando a ciência e a tecnologia de alimentos. A utilização de marcadores químicos para as fases da reação, bem como a avaliação dos impactos tecnológicos e nutricionais derivados desta reação devem estar cada vez mais presentes à realidade industrial e ao desenvolvimento de produtos lácteos.

\section{AGRADECIMENTOS}

Os autores agradecem ao CNPq e a FAPEMIG pelo suporte financeiro a projetos na área de produtos lácteos concentrados e desidratados, e, a CAPES e ao CNPq pelas bolsas de pós-doutorado e de produtividade concedidas.

\section{REFERÊNCIAS}

ANDRADE, C. D. Maillard reaction products: some considerations on their health effects. Clinical Chemistry and Laboratory Medicine, v. 52, n. 1, p. 53-60, 2014.

ARENA, S. et al. A. Dairy products and the Maillard reaction: A promising future for extensive food characterization by integrated proteomics studies. Food Chemistry, v. 219, p. 477-489, 2017.
BARBOSA, R. M. S. et al. Food intake by children based on the Brazilian food guide pyramid for young children. Revista de $\mathbf{N u}$ trição, v.18, n. 5, p. 633-641, 2005.

BASTOS, D. H. M. et al. Produtos da reação de Maillard em alimentos industrializados. Nutrire, v. 36, n. 3, p. 63-78, 2011.

BRIÃO, V. B. et al. Cinética do escurecimeno não-enzimático com soluções modelo de açúcares e aminoácidos em pH neutro e ácido. Acta Scientiarum Technology, v. 33, n. 1, p. 87-93, 2011.

Centro Nacional de Informações sobre Biotecnologia (NCBI). Composto de banco de dados PubChem, CID=237332. 2005. Disponível em: $<$ https://pubchem.ncbi.nlm.nih. gov/compound/237332>. Acesso em: 11 jan. 2016.

FENNEMA, O. R.; SRINIVASAN D.; KIRK, L. P. Química de Alimentos de Fennema. Porto Alegre: Artmed, 2010. 900p.

FRANCISQUINI, J. A. et al. Avaliação da intensidade da reação de Maillard, de atributos físico-químicos e análise de textura em doce de leite. Revista Ceres, Viçosa, v. 63, n. 5, p. 589-592, 2016.

GUERRA HERNÁNDEZ, E. et al. Chemical changes in powdered infant formulas during storage. International Journal of Dairy Technology, v. 55, n. 4, p. 171-176, 2002.

HELLWIG, M. et al. Stability of individual maillard reaction products in the presence of the human colonic microbiota. Journal of Agricultural and Food Chemistry, v. 63, p. 6723-6730, 2015.

KAVOUSI, P. et al. Formation and reduction of 5-hydroxymethylfurfural at frying tem- 
perature in model system as a function of amino acid and sugar composition. Food Chemistry, n. 182, p. 164-170, 2015.

KEENEY, M.; BASSETE, R. Detection of intermediate compounds in the early stages of browning reaction in milk products. Journal of Dairy Science, v. 42, n. 6, p. 945-961, 1959.

KELLOW, N. J.; COUGHLAN, M. T. Effect of diet-derived advanced glycation end products on inflammation. Nutrition Reviews, v. 73, n. 11, p. 737-759, 2015.

LE, T. T. et al. Direct evidence for the role of Maillard reaction products in protein cross-linking in milk powder during storage. International Dairy Journal, v. 31, n. 2, p. 83-91, 2013.

LECHNER, R. Analytische Abgrenzung: Pasteurisierte Milch H-Milch, Sterilmilch. Deutsche Milch, v. 33, n. 14, p. 489-494, 1982.

LEIVA, G. E., NARANJO, G. B., MALEC, L. S. A study of different indicators of Maillard reaction with whey proteins and different carbohydrates under adverse storage conditions. Food Chemistry, 215, p. 410416, 2017.

LIU, Q. et al. Physicochemical and antioxidant properties of Maillard reaction products formed by heating whey protein isolate and reducing sugars. International Journal of Dairy Technology, v. 67, n. 2, p. 220-228, 2014.

MALEC, LAURA S., et al. Loss of available lysine during processing of different dulce de leche formulations. International Journal of Dairy Technology, v. 58, n. 3, p. 164-168, 2005.
McSWEENEY, P. L. H. e FOX, P. F. Advanced Dairy Chemistry Lactose, Water, Salts and Minor Constituents. University College, Cork, Ireland: Springer Science Business Media. 2009.

MEHTA, B. M.; DEETH, H. C. Blocked Lysine in Dairy Products: Formation, Occurrence, Analysis, and Nutritional Implications. Comprehensive Reviews in Food Science and Food Safety, v. 15, 2016.

MONARO, E. L. F. Inibição da Formação de produtos da reação de Maillard por extrato de erva-mate (Ilex paraguariensis) em sistemas modelo alimento. 2012. 102 p. Dissertação (Mestrado em Nutrição em Saúde Pública) - Universidade de São Paulo, São Paulo, 2012.

O'CALlaghan, D. J., HOGAN, S. A. The physical nature of stickiness in the spray drying of dairy products: a review. Dairy Science \& Technology, v. 93, p. 331-346, 2013.

POULSEN, M. W. et al. Advanced glycation endproducts in food and their effects on health. Food and Chemical Toxicology, v. 60, p. 10-37, 2013.

RAJCHL, A. et al. M. Rapid determination of 5-hydroxymethylfurfural by DART ionization with time-of-flight mass spectrometry. Analytical and Bioanalytical Chemistry, n. 405, p. 4737-4745, 2013.

RODRIGUEZ, A. et al. Study of the browning and gelation kinetics in a concentrated sheep milk and sucrose system. International Journal of Dairy Technology, v. 69, p. 1-7, 2016.

ROSATELLA, A. A. et al. 5-Hydroxymethylfurfural (HMF) as a building block 
platform: Biological properties, synthesis and synthetic applications. Green Chemistry, v. 13, p. 754-793, 2011.

SAKKAS, L. et al. Assessment of heat treatment of various types of Milk. Food Chemistry, v. 159, p. 293-301, 2014.

SCHAMBERGER, G. P., LABUZA, T. P. Effect of green tea flavonoids on Maillard browning in UHT milk. Food Science and Technology, v. 40, n. 8, p. 1410-1417, 2007.

SCHUCK, P. et al. Recent advances in spray drying relevant to dairy industry: A comprehensive critical review. Drying Technology, in press, 2016.

SITHOLE, R., MCDANIEL, M. R., GODDIK, L. M. Rate of Maillard browning in sweet whey powder. Journal of Dairy Science, v. 88, n. 5, p. 1636-1645, 2005.

TROISE, A. D. et al. Evolution of protein bound Maillard reaction end-products and free Amadori compounds in low lactose milk in presence of fructosamine oxidase I. Food Chemistry, 212, p. 722-729, 2016.

URIBARRI, J. et al. Advanced glycation end products in foods and a practical guide to their reduction in the diet. Journal of the American Dietetic Association, v. 110, n. 6, p. 911-916, 2010.

VHANGANI, L. N.; WYK, J. V. Antioxidant activity of Maillard reaction products (MRPs) in a lipid-rich model system. Food Chemistry, n. 208, p. 301-308, 2016.

VISTOLI, G. et al. Advanced glycoxidation and lipoxidation end products (AGEs and ALEs): an overview of their mechanisms of formation. Informa Healthcare, v. 47, n. 1, p. 3-27, 2013.

VLASARA, H. et al. Advanced glycation end product homeostasis. Academy of Sciences, v. 1126, p. 46-52, 2008. 